\title{
Projective identification and
} tite tolong: "the unconscious defences against anxiety: okaschanged? social work education, practice learning, and the fear of failure

\author{
Jo Finch \& Jason Schaub
}

\begin{abstract}
ocial work and social work education have long been subject to rapid change and reform, including organizational and legislative reform, often in response to highly publicized (and politicized) failures in child protection policy and practice. Public Inquiries and Serious Case Reviews into deaths of young children at the hands of their parents and carers, for example, frequently produce reports highly critical of social workers, and to a lesser extent other professionals, for the many missed opportunities that, with the benefit of hindsight, might have resulted in a less tragic outcome for the child concerned. Such reports often stress poor information-sharing and communication between professionals from different organizations, the need for appropriate supervision and scrutiny, and an inability to focus on the needs of the child.

In the wake of widely publicized child deaths-for example, Victoria Climbié and Peter Connelly (both in the London Borough of Haringey) - the last eleven years in England has seen major policy shifts in both social work provision and social work education, leading to what one social work academic aptly described to us as the "homogenization" of social work programmes across England. This process of homogenization has developed in an accompanying political climate of performance management, targets, inspection regimes, audit cultures,
\end{abstract}


risk-averse practice, and a sanitizing technical-rational approach to theaverse: ok? task of social work-what Cooper and Lees refer to in chapter 15 as the dominance and pervasiveness of "New Public Management" theory, which has had a profound effect on welfare organizations.

Within the context of a rapidly changing social work culture under threat by the neo-liberal enterprise, this chapter centres on an important aspect of social work education in England: practice placements. The discussion explores the complex and challenging dynamics that are created between practice educators (social workers tasked with the job of assessing students on placement) and social work students. In our roles as social worker lecturers (formerly social workers in children and family settings), we were curious about why working with struggling or failing students appeared to prove such a difficult emotional experience for practice educators, particularly those working in children and family settings. We also noted how challenging it was for social work tutors. Additionally, we felt that the existing research base offered little in the way of a satisfactory explanation (for a thematic review of the literature, see Finch \& Poletti, 2013; Finch \& Taylor, 2013). Rather, the reasons proffered seemed defended in themselves, and the impact of affect was notably absent.

We were able to explore in an earlier discussion (Finch, Schaub, \& Dalrymple, 2014) how far the concept of projective identification was useful in understanding why practice educators appeared to find the experience of failing a student so painful. We argued that these multifarious dynamics came sharply to the fore when students were underperforming or, indeed, failing their placements. One possible and very serious consequence of those complex dynamics, which appeared to powerfully invoke strong, uncomfortable, and intolerable emotions, was that practice educators may be reluctant, or indeed unable, to fail students when required.

We also noted in our empirical work potential concerns about variations in practice in different fields of social work. For example, practice educators within children and family settings appeared to have heightened levels of emotionality and difficulties around managing and reconciling the role of practice educator, and they seemed more reluctant to fail students in comparison with, for example, practice educators in mental health settings.

What we had not explored in the analysis was the dynamic between these individually felt projections and the organizational and societal context; as such, there was a missed opportunity to explore how far 
such projective-identification processes were symptomatic of wider organizational and societal defences against anxiety. This raises the question of the possible cause for this anxiety, which this chapter also explores.

We have the opportunity now, therefore, to consider these questions further to see if we can make a case to consider these powerful projective identifications that emerged so starkly in our research as:

1. a specific and powerful individual manifestation of an unconscious social defence against anxiety, which emerges most blatantly, we argue, when students are underperforming or indeed failing in practice learning settings;

2. persecutory psychic processes that induce anxiety states in practice educators;

3. the inducement, by the latter anxious states, of defensive practices that include being mobilized to not be able to think, reflect, and act appropriately.

We aim, therefore, to consider further the nature of these anxieties in social work, which, like Menzies Lyth's study, highlighted anxiety arising from the primary task-namely, the "continuous care for patients, day and night, all the year round" (1960, p. 97). It is possible that they emerge from the primary anxieties proffered by users of social work services. We suggest that these anxieties are also symptomatic of wider societal concerns and preoccupations about the often unbearable task of social work, one of which is the pervasive fear of failure. The exercise of further analysis and theorization of our research data therefore has the potential to build upon Menzies Lyth's original unconscious defences against anxiety proposition, by using contemporaneous research in a specific professional context-the social work practice placement.

The chapter begins with an overview of the changes and developments in social work and social work education, clearly very different to the context and culture of nurse training in the late 1950s-although yet sharing perhaps some of the complexities and emotional pain observed so acutely by Menzies Lyth. There is then a brief discussion of our previous empirical work in this area. The chapter next focuses on projective identification before going on to document how projective identificatory processes emerged in our research studies. There is then a discussion of the context of wider social work anxieties, which, we argue, impact upon and are played out by practice educators and 
social work students. The chapter finishes with a brief discussion about containment and, in light of our discussion, considers possible ways forward.

\section{Social work education}

The importance of practice learning on UK qualifying social work programmes, both undergraduate and post-graduate, has been emphasized by the plethora of reform in both social work and social work education over the last 11 years. The introduction of the degree in social work in 2003, for example, increased the number of assessed days in practice from 130 to 200. Students were required to undertake two, sometimes three, placements in different settings and to have the opportunity to undertake statutory tasks and interventions. Students were assessed against newly implemented national occupational standards for social work. Social work became a protected professional title, and four regional care councils were set up to regulate social work and social work education. Programmes' suitability processes were strengthened, and students were required to demonstrate they were "fit for practice" before beginning their placement. The purported aim of these reforms was to increase public trust and confidence in the social work profession, to ensure parity with comparator professions (e.g., nursing), and to transform its status and image (DoH, 2002; Orme et al., 2009). These reforms can be seen as a consistently anxious policy response to the "problem" of social work, most notably its apparent constant failure to intervene appropriately in cases of child abuse.

More reform was enacted following the Social Work Taskforce (SWTF) report in 2009, set up in the wake of political, media, and public outcry at the death of a young child, Peter Connelly, who was killed by his carers (SWTF, 2009). The SWTF's later iteration, The Social Work Reform Board, continued the developmental work recommended, which included the setting up of a College of Social Work. Other changes included the abolition of the General Social Care Council, the regulatory body for social work in England, with the functions now being managed by the Health Care Professions Council (HCPC); the adoption of a professional-capability framework to assess practice learning; and a two-part qualification now required for practice educators assessing final-year students.

There have also been a number of reviews into the practice of social workers. The Laming Report focused on developments since Victoria 
Climbié (Laming, 2009), and the Munro review focused on children and family social work (Munro, 2011b). The Munro review documented the unintended effects of the growing trend towards managerialism in social work, with the accompanying focus on targets having consequences in terms of professional judgement, autonomy, and practice wisdom. Most recently, Narey (2014) and Croisdale-Appleby (2014) have each recommended further changes to qualifying social work programmes. Croisdale-Appleby makes a number of recommendations, including moving training away from undergraduate level to postgraduate level and developing yet more stringent entry requirements and matching workforce development needs with the numbers of trainees. Narey adopts a more critical stance, advocating a move back to specialist routes in qualifying training away from the current generic model - completely at odds, of course, with the HCPC's and the College of Social Work's generic endorsement standards. Alongside these reviews (the former commissioned by the Department of Health and the latter by the Department of Education), a new children and families social work training scheme called "Frontline", similar in nature to the "Teach First" model, is due to be rolled out in September 2014, subject to HCPC approval. It is notable that the programme is not seeking endorsement from the College of Social Work. At the time of writing, a similar scheme in mental health, termed "Think Ahead", has also been announced. Thus, the constant threat and culture of continuous change is still very potent within social work education, possibly impacting on academics, students, and other key stakeholders within local authorities. These different reviews take place in the context of a difficult economic climate, huge cuts to welfare expenditure, draconian housing policies (such as the "bedroom tax"), and the continued demonization of recipients of welfare.

Despite the flurry of reforms within social work education and reforms to practice learning, there have been persistent concerns raised about the placement component on social work programmes. Finch and Taylor (2013) identified historical and continuing concerns centred around three areas:

1. the quantity and quality of placements;

2. the low failure rate;

3. the apparent reluctance of practice educators to fail students.

In terms of failure rates, for example, Menzies Lyth noted a high attrition rate for student nurses in the hospital under study, approximately 
one-third, and it is interesting to note that concerns about high attrition rates on nursing programmes remain a contemporary concern (Gidman, 2001). Indeed, the attrition rate in nursing education in 2003 was estimated to be $19 \%$ nationally (Glossop, 2002). This situation contrasts significantly with social work in England, as well as in other countries, which has relatively low failure and withdrawal rates (Finch \& Taylor, 2013; Hussein, Moriarty, Manthorpe, \& Huxley, 2008). Indeed, despite a number of changes to the qualification requirements over the years, as well as significant changes in entrance requirements, the failure rate has remained constant at around 2-3\% (Finch \& Taylor, 2013). The SWTF (2009) interim report, pertinently titled, Facing Up to the Task, stated ominously:

Specific concerns have been raised about the ... robustness and quality of assessment, with some students passing the social work degree who are not competent or suitable to practise on the frontline. [SWTF, 2009, p. 24]

Narey and Croisdale-Appleby also raised concerns about practice learning. Narey claims that placements are often poor quality and in non-statutory settings, and they do not prepare students adequately for the task of statutory children and families social work. CroisdaleAppleby noted that the quality of placements was inconsistent. From these different origins, it is clear that the placement element of social work training poses distinct challenges and contradictions. It lies at the intersection of social work's reality and the theoretical and abstract ideals of the academy, yet it remains a problematic site for many reasons.

In addition to these concerns, social work remains a contested activity. Therefore, assessing what is "good-enough" social work practice poses distinct challenges, not least in a culture where social work (and, by association, social work education) is often deemed to be "not good enough"; while low failure rates on their own do not necessarily indicate a failure to fail by the social work education system, they certainly are indicative of an anxiety around failing. Unlike the nursing education system of the 1950s, social work students are not voluntarily withdrawing.

\section{Preceding studies}

Our previously undertaken empirical work focused largely on practice educators' experiences of working with failing students. Finch's (2010) doctoral study examined why it seemed so hard for practice educators 
to fail social work students, and it highlighted the emotional pain associated with failing a student, which at times, it was argued, prevented practice educators from failing students when the evidence appeared to be unequivocal and was highly indicative of failure. Schaub and Dal-

near repetition deliberate, or should this sentence be combined with the previous one (by adding "and acute anxiety" after "emotional distress")?
Finch in press: published? rymple's study $(2011,2013)$, while initially aiming to consider the support needs of practice educators, nonetheless identified the significant emotional distress experienced by practice educators when working with struggling or failing students. These studies also noted the acute anxiety felt by practice educators when confronted with a struggling or failing student.

Our work has since developed: first, through undertaking a comparative study with Italian practice educators, which gave us the opportunity, among other things, to consider more critically the English context, not least the unintended effect of the assessment framework and the particular culture of English social work (Finch \& Poletti, 2014). Second, further empirical work was undertaken to consider other stakeholders, namely social work tutors (Finch, in press) and important key sites of group decision-making about failing (and passing) social work students (Finch, 2013); and third, by extending our theoretical analyses further. For example, we considered how far the concept of projective identification was helpful in explaining these powerful feelings, which typically included, anger, rage, anxiety, and guilt and the subsequent ways in which practice educators were mobilized to act out or voice these projections (Finch, Schaub, \& Dalrymple, 2014).

\section{Projective identification}

Building on the ideas of Freud, Klein developed the concept of projective identification. The concept was later taken up by Bion and others within the objection relations tradition. Projective identification can be seen as an unconscious defensive strategy that aims to protect from psychological attack or harm. We argued elsewhere (Finch, Schaub, \& Dalrymple, 2014) that given the heavily debated nature of the concept, projective identification can perhaps be least controversially conceptualized as a mode of unconscious communication of emotion. Projective identification, however, is more complex than a transference from one person to another; rather, it is often an expulsion of "unwanted or threatening ideas" (Frosh, 2012, p. 162), ranging from the relatively benign to the much more threatening and hostile. Projective-identification processes thus occur when a person cannot bear or tolerate certain 
aspects of him/herself and projects these deeply unwholesome and unsettling emotional states that cannot be borne into another person. These projections are often so powerful that they compel the object of the projections to mobilize those unconscious feelings into actions or behaviours and may impact on their ability to reflect, think, and act appropriately (Trevithick, 2011).

Projective-identification processes, then, are usually viewed as a dynamic between two people, occurring in all relational contexts, not just within a therapy room. We contended, therefore, that when confronted with the spectre of failing a placement-and often alongside that, failing the programme-a student is likely to be in distress and experiencing a range of conflicting emotions. Some of these will be conscious, but the fear of failure, of not being "good enough", and the accompanying feelings of guilt and shame, are likely to provoke deeply held unconscious feelings, emanating from infancy and childhood, that are intolerable for a student to bear. The practice educator becomes the object into which these deeply unsettling emotional states of mind are projected. There may also be envious feelings in the untenable and uncomfortable mix, such as an unconscious desire by the student to enter "the mind of the other in order to acquire the desired aspects of his psyche" (Spillius, Milton, Garvey, \& Couve, 2011, p. 126). Indeed, Klein stated that projective-identification processes from the infant to the mother aimed not only at ridding himself of his bad parts but also "take control": should intended to injure, take control, and possess the mother (Klein, 1952). .0 f'? $^{2}$ ? Klein later noted the envious nature of much projective-identification something missing, processes (Klein, 1957), which we feel is of particular relevance to prac-or delete "much"? tice educator-social work student interactions.

\section{Projective identification in action}

We noted in our analysis of the data from across the studies that practice educators often appeared mobilized by the student's powerful projections, in a number of ways. First, by experiencing extremes of emotion, including intense anxiety; second, by referring at times to students (and universities) in unprofessional, hostile, and blaming ways; and third, by being unable to think, reflect upon, or contain such feelings or indeed use them to critically consider the student's state of mind and what was being communicated. These projections were so unsettling that sometimes the results were abruptly ended placements, poor assessment reports, or collusion. The latter some- 
sentence added to explain who the people quoted below are, otherwise it's not immediately clear who "Lily" etc. are. Sentence ok as worded?

times took the form of avoiding the task of failing the student-either not addressing concerns quickly enough, making it subsequently difficult to uphold the failed decision because due process had not been followed, or, of more concern, failing to fail the student. We make the case, therefore, that the reluctance or inability to fail a student may well be a form of defensive response to these persecutory projections that induce an anxious state. The narratives of practice educators below illustrate this.

In what we considered to be almost paralysing levels of painful emotion, Lily stated:

I was incredibly confident with the successful engaged students but with the difficult student my confidence levels went down a lot... I was anxious ... I was worried.

Jennifer recognized she had become part of a dynamic with the student where she "felt like I was working harder than him in his practice placement" yet could not escape from this rescuing dynamic. She stated:

... he [student] took on the role of a child sometimes and puppy dog. I'm the weak one and you're the strong one and it will be in your hands and you're the supervisor ... [it] carried on, the puppy dog eyes.

This caused Jennifer to experience anger. She stated:

I got angry with him sometimes. I wasn't angry at him, I was angry at home, I would be smouldering, pissed off ... .

Anger was seen in other narratives, and, in response to the anger felt, practice educators also experienced guilt. Martha reported feeling "terribly guilty", and Daisy stated:

... because at the time I made that decision, the guilt, it was unbearable... it was a reality check ... oh my god, what about her children ... I felt like I am a rotten shit. from a professional discourse, which revealed itself in angry and personal comments about the students. For example, Lily described the student as: 
... absolutely terrible, she was appalling, she was abysmal and no way should she ever be near clients ... there were a million difficulties with her...

This was seen most starkly in Daisy's narrative, which was shocking, full of profanity, and extreme:

... I just thought ... I thought, "Fuck you! You are not going to apologize for your fucking behaviour with a period. Every fucking woman in the world gets a period, yes some have difficulties, some get emotional. ... You've like resorted to like fucking bottom of the barrel ..."

Daisy makes hurtful and personal comments about the student's body size (the student was significantly overweight) and fantasizes about service users being abusive to the student. She states:

... they'll call you a fat bitch because you are fat ... it will be their way of releasing, of hurting you.

Some practice educators articulated the persecutory projections in terms of feeling threatened by the students. Mary, for example, stated:

At the end, when he didn't complete what he was to have completed, I explained, again, that I was going to fail him, and he became sort of aggressive, you know that sort of silent aggression? Intimidation like, you know, what are you doing failing me? It was very unpleasant in his reaction to me.

Carla also expressed fears about what she termed, "the student making damaging claims". She stated:

It felt like, even my colleague said to me, "She's dangerous, she's going to come in here and wreck somebody's career." Somebody could work their way up for years, and she could come in and say something, and that would be the end of their career. Tons of people just didn't trust her being around.

Lily, a very experience practice educator of both nursing and social work students, described a student who to "put the fear of God into me" and recognized that the student: 
... beat me down really with threats and I allowed myself to be beaten down.

Lily acknowledged that:

It was the worst career decision I have ever made, and to this day, I have a huge regret about it.

This leads to the concern that such projective identificatory processes thus mobilize practice educators to act out, and a defensive mechanism emerges in turn whereby practice educators cannot fail students. It appeared to us that to fail the student was akin to acknowledging practice educators' own failures. Indeed, Terry stated:

That was the thing I was struggling with through the whole thing. How much of her failure was a reflection of my own practice teaching?

Terry carried on with this theme:

I would say probably $90 \%$ of the time, if the student fails there's something wrong with the practice teacher. of sentence?)

We also noted that in the retelling of the stories in the research-interview context, the use of present-tense language to explain a past event, so that it appeared to us that even in the narrating of the story the practice educators were still mobilized and affected by these projections. It is important to note, however, that not all practice educators expressed the same level of emotional distress, and there was evidence of practice educators managing to fail students in an emotionally contained and professional manner.

\section{Social work anxieties}

Defences against anxiety and pain, although often felt to be an individual concern, are also developed by institutions to protect against threats. These threats thus arise from a range of possible sources, both external and internal. Externally these threats may include changes in governmental policy, legislative change (often in response to moral panics), and other social change (Halton, 1994). Internally these threats might concern poor employee relations and, more often, the type of 
work the organization is engaged in and who it works with. Defensive strategies thus emerge in a complex dynamic interplay between individuals and organizations. Given all the continuous reforms within social work as discussed earlier, it is not surprising that anxiety within social work is high, coupled with the anxieties than emanate from service users and the contested nature of the "primary task" - that is, care and control.

The question then arises as to the nature of the anxieties that affect the social work training system more generally and how these both effect and are symptomatic of the practice educator-student relationship. Perhaps a further pertinent, related question is: Why are they brought to the fore so acutely when issues of failing students emerge? We contend that there are two reasons for this: first, the deeply held unconscious fear of failure, felt by us all; and second, the public perception of social work as a "failing profession" - in short that we cannot get our primary task right, namely to adequately safeguard and protect children from harm. This is made all the more acute and, importantly, public by inspection regimes, audit cultures, performance management, and blaming Inquiry and Serious Case Review Reports.add Public"? Alongside this, social work is imbued with public and political anxiety that emerges so vehemently when a child dies at the hands of her or his parents-namely, because social work has failed to prevent it coming to the public's consciousness (Cooper \& Lousada, 2005). The response then, often in the form of policy change but intense criticismsomething is wrong of social work, is often hostile, attacking, and blaming, resulting in the here construction of further defensive practices and states of mind, which, as so acutely observed in Menzies Lyth's study, may well be obstructing the primary task.

It is not surprising to us, then, that the practice educators most struggling with their task were those in children and family settings. Indeed, the care-versus-control function inherent in social work, which, as Evans and Harris (2004) note, places contradictory demands on social workers, appeared to be played out in practice educator-student relationships via the conflict felt between the nurturer and enabler-oflearning role, versus the assessor role. Lily was able, to some extent, to acknowledge this; she stated:

... there was almost like a maternal feeling about the facilitation of learning but the flipside of that was when I had to become the kind of teller-off or the person who was making judgements ... I did struggle with that. 
This contrasted with an account of a mental health social worker, who, in discussing the practice educator role, stated:

... you know it's meant to be a mature student I am dealing with you know, we are entering into this arrangement right, as adults, you know I had my part to play, the student has their part to play ... I just kind of felt, well, this [failing a student] is going to happen in practice placement . . . this is primarily your responsibility, it's your responsibility at the beginning as well, to think about what you're getting into.

\section{Containment}

As Menzies Lyth's work demonstrated so starkly, organizational defences arose in the hospital, which proved unhealthy and psychologically damaging, resulting in poor-quality care of patients, poor inter-relations, high sickness levels, and high levels of attrition. For the

between who? perhaps change rest of sentence to: "poor nurse-patient relations, and high levels of sickness and attrition rates among nurses" practice educators in our studies, they reported that the experience of working with a failing student was stressful, that they felt unsupported by the university and isolated, and that at times they felt threatened by the student. The experience of working with a failing student can all too often leave a lasting legacy on the individual practice educator as well as on the team. Indeed, several practice educators in our studies decided not to take on any further students. The crucial question is, how do practice educators cease to be mobilized, but, instead, make sense of and reflect upon what is being so forcefully communicated?

It thus seems patent to us from our research into practice learning and subsequent theoretical analysis that containment is key to minimizing the negative and damaging impact of projective identification. While social work will always remain an anxiety-infused profession, finding ways to contain these anxieties is crucial. We saw in our studies missed opportunities for practice educators to experience the projections as a form of communication, which could have served to help students make sense of and articulate their own anxieties, psychological processes, and states of mind. The use of Ruch's (2007) work in terms of containment within children and family social work settings, for example, would equally be applicable to practice education. She argues for the need for "safe" spaces that would allow social workers, or in this case practice educators, to explore uncomfortable and difficult emotions to "make sense of the uncertainty and anxiety they encounter on an everyday basis" (p. 662). 
As stated earlier, not all practice educators involved in our studies experienced the paralysing levels of emotional pain, although it was still noted as an unpleasant experience. We noted that those practice educators, the majority of whom were, or had been, approved social workers (now called approved mental health practitioners),

1. did not experience any role conflict or strain between the nurturer or enabler of learning and the assessor role of their function;

2 saw failing students as an inevitable part of the job;

3. saw the experience as a learning one;

4. could clearly articulate their responsibilities and boundaries as a gatekeeper to the profession and as a practice educator;

5. saw the student as an adult learner with clear responsibilities;

6. did not internalize the students' failure as their own.

This suggests that the anxieties within social work and practice education can be managed and that projective identificatory mechanisms can be appropriately accommodated, contained, and withstood. This points to a way forward in light of findings, although further research into the psychic strategies employed by such practice educators to withstand the projections of failing students is clearly indicated.

\section{Conclusion}

We have argued, therefore, that within social work and social work education there are multiple sources of anxiety and that there is a powerful reinforcing interaction between individuals' every day, lived experiences of engaging in unconscious defences strategies, as well as organizationally constructed social defences. These interactions operate within a distinct societal, political, and ideological context. Our research to date has thus focused on a particular manifestation of an unconscious defence against anxiety, and we have attempted to use our contemporary research on the issue of assessing failing social work students in practice learning to identify, first, another site where Menzies Lyth's proposition of unconscious defences against anxiety is relevant and, second, how such psychic processes impact on practice educators.

To finish on a reflective note, writing this chapter has been a challenge for us-that is, as social work lecturers who are relatively new to psychoanalytic theory, and are heavily imbued with sociological theories, we were also assailed by fears of "not being good enough". At the 
same time, we are working in a culture where we are also subject, at times, to the same persecutory and envious projections from students (as well as practice educators) and are sensitive to the same social work anxieties. Such an exploration reminds us starkly of these anxieties. It was interesting to note our own difficulties in thinking when both immersing ourselves in the data and constructing this chapter, as well as our anxious responses. 